\title{
ON THE POSSIBILITY OF FINDING LIGHT UNCOLORED SUPERSYMMETRIC PARTNERS AT PRESENT AND FUTURE MACHINES
}

\author{
J.-M. FRÈRE and G.L. KANE \\ Randall Laboratory of Physics, University of Michigan, Ann Arbor, MI 48109, USA
}

Received 21 March 1983

\begin{abstract}
Supersymmetric models allow the possibility of finding new light spin $-\frac{1}{2}$ fermions $\left(m<m_{\mathrm{W}}\right.$, and perhaps $m<10-15 \mathrm{GeV}$, charged or neutral) that are (apart from mixing effects) the supersymmetric partners of $\mathrm{W}, \mathrm{Z}^{\mathrm{O}}$, and Higgs bosons. We provide a detailed analysis of their expected properties, production mechanisms, and signatures, with emphasis on detection at $\mathrm{e}^{+} \mathrm{e}^{-}$colliders. Although the charged, spin $-\frac{1}{2}$ particles resemble sequential leptons, it turns out that their properties differ enough that they might have been missed in the standard searches with normal cuts, and they still might be found with $m<18 \mathrm{GeV}$. A neutral, spin- $-\frac{1}{2}$ particle with $m$ below about $30 \mathrm{GeV}$ could exist with a clear decay signature and be singly produced at detectable rates at present machines (picobarn cross sections).
\end{abstract}

\section{Introduction}

There are at present no experimental indications of which direction particle physics should go in order for us to better understand the Standard Model and why it works, the meaning of the apparent need for scalar particles in the standard model, and other current questions. Many theorists consider it possible that the next stage will be a supersymmetric ${ }^{\star}$, and/or grand unified gauge theory. One way to discover if supersymmetry holds in nature is to search for the supersymmetric partners of conventional particles.

In a supersymmetric theory, there will be partners for quarks, gluons, leptons, etc. Since such particles obviously do not occur with the same masses as their partners, the supersymmetry is broken. The masses which the supersymmetric partners are expected to have are then dependent on the (presently unknown) scale of supersymmetry breaking, and vary from model to model. There is no general pattern of masses, but typically scalar-quarks and scalar-leptons have masses of order $m_{\mathrm{W}}$. Gluinos are often light $(1-30 \mathrm{GeV})$ but can be much heavier.

In this paper we will discuss in detail how to look for the spin-1 $\frac{1}{2}$ partners of Higgs bosons and of $\mathrm{W}^{ \pm}, \mathrm{Z}^{0}$. It is necessary in a supersymmetric theory to have two (or more) doublets of Higgs ${ }^{\star \star}$ bosons, so physical charged Higgs arise. The fermionHiggs thus come in two weak interaction doublets, with particles $\tilde{\mathrm{H}}_{1}^{+}, \tilde{\mathrm{H}}_{1}^{0} ; \tilde{\mathrm{H}}_{2}^{0}, \tilde{\mathrm{H}}_{2}^{-}$.

* For a recent review and a place to trace the literature, see ref. [1].

$\star \star$ We will use the expression "Higgs particles" as a shorthand for $\mathrm{BEGH}^{2} \mathrm{~K}$ bosons. 
The partners of $W^{ \pm}$and $Z^{0}, \tilde{W}^{ \pm}$and $\tilde{Z}^{0}$ (the weak gauginos), can mix with the fermion-Higgs, and with the photino as well. Although we will use models for examples, we will give a general discussion of the phenomenology of the spin- $\frac{1}{2}$ supersymmetric partners, for both present and future machines. Some discussion has already been given by Ellis and Ross (2).

In many models a number of these particles come out quite light, so they are good candidates for which to look. A positive experimental result would, of course, be of extraordinary importance. On the other hand, a negative result using presently available machines, while it would constrain thinking in a helpful way, generally does not eliminate any ideas. When colliders with $\sqrt{s} \simeq m_{\mathrm{Z}}$ are available stronger statements will be possible; this is because most approaches which attempt to explain the weak scale (and thus $m_{\mathrm{W}}, m_{\mathrm{Z}}$ ) will produce some fermion-Higgs and gauginos that are lighter than $m_{\mathrm{w}}$. Some charged and some neutral states usually come out to be a few $\mathrm{GeV}$ in models.

There may be one situation where stronger statements are possible. In a very large class of models the light neutral fermion-Higgs will have masses and decay signatures which allow their production and detection at $\mathrm{e}^{+} \mathrm{e}^{-}$colliders at presently available energies and with $\mathscr{L} \geqslant 10^{31} / \mathrm{cm}^{2} \mathrm{sec}$. We will describe this in detail in sect. 4 below. If these states are absent it will seriously affect current theoretical efforts.

One suprising result is that the natural observation that a charged fermion-Higgs is similar to a sequential lepton, and thus experimentally excluded for masses up to about $18 \mathrm{GeV}$ by PETRA/PEP experiments, may not hold in practice. the reason is essentially that the supersymmetric particles which interact rather weakly and escape detectors are often massive, and the remaining leptons or jets may not have sufficient energy to survive usual experimental cuts (right-handed couplings often enter and further soften the lepton spectra).

The actual mass eigenstates produced are mixtures of weak eigenstates in interesting ways; we will discuss them below. We will describe in the following the production properties and the decay signatures of all the states which might be light enough to be observed. Some of what we discuss has been considered, sometimes generally and sometimes in specific models, by Ellis and Ross [2]; Arnowitt et al. [3]; Weinberg [4]; Ibáñez [5]; and Alvarez-Gaumé et al. [6].

In sect. 2 we try to provide a helpful notation, and carefully distinguish weak and mass eigenstates. We discuss the expected spectrum of particles and their quantum numbers, write mass terms, mass matrices, and interaction lagrangians. Sect. 3 analyses the decay possibilities for the charged states in a general way, categorizing most (all?) models, and explains how these states might so far have escaped detection at present machines. Sect. 5 provides analysis for the neutral states. Our notation is summarized in tables $1-3$. Someone who is interested mainly in experimental aspects can skip sect. 2 , using tables $1-3$ plus sects. 3,4 . 
TABLE 1

\section{Rules}

(1) Supersymmetric partners have a $\sim$.

(2) Weak interaction eigenstates are denoted by mnemonic capital letters, such as $\tilde{W}, \tilde{Z}, \tilde{B}, \tilde{H}$.

(3) Mass eigenstates are denoted by mnemonic lower case letters, such as $\tilde{w}, \tilde{z}, \tilde{h}$. Note that the couplings of a mass eigenstate might not be those suggested by its name; e.g. $\tilde{h}^{+}$may be a mixture of $\tilde{H}^{+}$ and $\tilde{W}^{+}$, the latter coupling proportional to the gauge coupling strength to any doublet, and the former coupling proportional to the mass of the interacting particles.

TABLE 2

$$
\begin{gathered}
S U(2) \text { eigenstates } \\
\tilde{W}^{ \pm}, \tilde{W}^{0}, \tilde{B}^{0}, \tilde{H}_{1}^{+}, \tilde{H}_{2}^{0} \\
\tilde{H}_{1}^{0}, \ldots \\
\tilde{H}_{2}^{-}, \ldots \\
S U(2) \times U(1) \text { eigenstates } \\
\tilde{W}^{ \pm}, \tilde{Z}^{0}, \tilde{\gamma}^{0}, \ldots \\
\text { Mass eigenstates } \\
\tilde{w}^{ \pm}, \tilde{z}^{\prime}, \tilde{\gamma}, \tilde{h}_{1}^{+}, \tilde{h}_{1}^{0 \prime}, \tilde{h}_{2}^{0 \prime}, \tilde{h}_{2}^{-}, \ldots
\end{gathered}
$$

Mass eigenstates after removing degeneracies in neutral sector

$$
\tilde{z}, \tilde{\gamma}_{1}, \tilde{h}^{n}, \tilde{\gamma}_{2}, \ldots
$$

\begin{tabular}{|c|c|c|}
\hline $\begin{array}{c}\text { Masses } \\
(\mathrm{GeV})\end{array}$ & Charged & Neutral \\
\hline 100 & $\cdots--\tilde{e}, \tilde{\mu}, \tilde{\tau}, \tilde{\mathrm{u}}, \tilde{\mathrm{d}}, \tilde{\mathrm{s}}, \tilde{\mathrm{c}}, \tilde{\mathrm{b}}, \tilde{\mathrm{t}}$ & $\cdots-\tilde{\nu}_{\mathrm{e}}, \tilde{\nu}_{\mu}, \tilde{\nu}_{\tau}$ \\
\hline $\begin{array}{c}M_{\mathrm{Z}} \\
80\end{array}$ & $\cdots--\tilde{w}^{+}$ & $---\tilde{z}^{0}$ \\
\hline 60 & & \\
\hline 40 & & $\ldots-.--\tilde{h}^{0}$ \\
\hline 20 & $\ldots-\bar{h}^{+}$ & $-\cdots--\tilde{g}$ \\
\hline 0 & & $\cdots-\dot{\gamma}_{2}$ \\
\hline
\end{tabular}

\section{Generic names}

$\bar{h}^{+}=$lightest charged mass eigenstate. Its couplings are model dependent; it might be mainly Higgs-like or comparable mixtures of fermion-Higgs and wino.

$\tilde{\gamma}_{1}, \tilde{\gamma}=$ light neutral mass eigenstates which are mixtures of photino and fermion-Higgs.

TABLE 3

Possible spectrum 


\section{Particles and lagrangians}

In this section we give the details of the particle interactions and mass matrices; readers mainly interested in connections with experiment may skip to sect. 3 .

We first proceed to write down the fermionic part of the interaction lagrangian. For reasons of convenience, we prefer to use the 2-component notation, and we outline below some simple conversion rules. The correspondence with 4-component notation is easily established in the Weyl basis, where the $\gamma$ matrices read:

$$
\begin{gathered}
\gamma^{0}=\left(\begin{array}{ll} 
& 1 \\
1 &
\end{array}\right), \quad \gamma^{i}=\left(\begin{array}{cc}
\bar{\sigma}^{i} & \sigma^{i}
\end{array}\right), \\
\left(\bar{\sigma}^{i}=-\sigma^{i} ; \bar{\sigma}^{0}=\sigma^{0}\right), \quad \gamma^{5}=\left(\begin{array}{cl}
-1 & \\
& 1
\end{array}\right) .
\end{gathered}
$$

The 2-component spinor $\psi$ may then be written $\frac{1}{2}\left(1-\gamma^{5}\right) \Psi$ or $\Psi_{\mathrm{L}}$, where $\Psi=\left(\begin{array}{c}\psi \\ \text { any }\end{array}\right)$. Introducing $\Lambda=\left(\begin{array}{c}\lambda \\ \text { any }\end{array}\right)$ we have

$$
V^{\mu}=\bar{\lambda} \bar{\sigma}^{\mu} \psi=\bar{\Lambda} \gamma^{\mu}\left(\frac{1-\gamma^{5}}{2}\right) \Psi,
$$

(note that in 2-component notation $\bar{\lambda} \equiv \lambda^{*}$ while in 4-component notation $\bar{\Psi}=$ $\left.\Psi^{+} \gamma^{0}\right)$.

We note that (for anticommuting operators)

$$
V^{\mu}=\bar{\psi} \bar{\sigma}^{\mu} \lambda=-\lambda \sigma^{\mu} \bar{\psi} .
$$

We may thus alternatively use other spinors

$$
\Psi^{\prime}=\left(\begin{array}{c}
\text { any } \\
\bar{\psi}
\end{array}\right), \quad \Lambda^{\prime}=\left(\begin{array}{c}
\text { any } \\
\bar{\lambda}
\end{array}\right),
$$

and write the interaction as:

$$
V^{\mu}=-\bar{\Lambda}^{\prime} \gamma^{\mu}\left(\frac{1+\gamma^{5}}{2}\right) \Psi^{\prime} .
$$

A scalar coupling, on the other hand, does not flip sign:

$$
\lambda \psi=\psi \lambda .
$$

The absence of complex conjugation in this term induces us to mix the representations above, and use in 4-component notation:

$$
\lambda \psi=\bar{\Lambda}^{\prime} \Psi_{\mathrm{L}}=\left(\frac{1+\gamma^{5}}{2} \Lambda^{\prime}\right)^{\dagger} \gamma^{0} \Psi_{\mathrm{L}}=\left(\bar{\Lambda}_{\mathrm{R}}^{\prime}\right) \Psi_{\mathrm{L}} .
$$

The above mentioned rules should allow us, wherever possible, to regroup fermions into 4-component Dirac particles. (An explicit example will be given below.) 
The particles we are essentially interested in are listed below, in "left-handed" notation.

Particles:

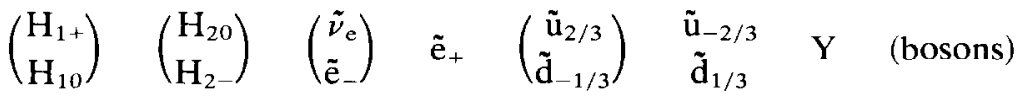

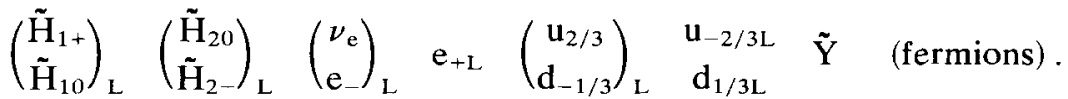

The doubling of the Higgs structure required by the supersymmetry is by now familiar; an $\mathrm{SU}(2) \times \mathrm{U}(1) \times \mathrm{SU}(3)$ singlet $Y$ and its partner $\tilde{Y}$ are often needed to avoid the appearance of an unwanted axion, so we include them as well. The usual repeating of generations is assumed.

The vector superfields of $\mathrm{SU}(2) \times \mathrm{U}(1)$ are taken either in the $\left(W_{i}, B\right)$ basis or in the $\left(W^{ \pm}, Z, \gamma\right)$ basis. Their components are both vector fields and 2-component fermions:

$$
\left(\begin{array}{cc}
W_{i}^{\mu} & B^{\mu} \\
\tilde{W}_{\mathrm{L} i} & \tilde{B}_{\mathrm{L}}
\end{array}\right), \quad \text { or } \quad\left(\begin{array}{ccc}
W_{ \pm}^{\mu} & Z_{0}^{\mu} & \gamma \\
\tilde{W}_{ \pm \mathrm{L}} & \tilde{Z}_{0 \mathrm{~L}} & \tilde{\gamma}_{\mathrm{L}}
\end{array}\right)
$$

Gauge couplings: in terms of weak eigenstates we list the relevant fermionic terms from the lagrangian (quark couplings are omitted since they strictly parallel leptonic interactions)

$$
\begin{aligned}
\mathscr{L}_{\mathrm{G}}= & \left(e / \cos \theta_{\mathrm{w}}\right) \frac{1}{2} B_{\mu}\left(\overline{\tilde{H}}_{1+} \bar{\sigma}^{\mu} \tilde{H}_{1+}+\overline{\tilde{H}}_{10} \bar{\sigma}^{\mu} \tilde{H}_{10}-\overline{\tilde{H}}_{2-} \bar{\sigma}^{\mu} \tilde{H}_{2-}\right. \\
& \left.-\overline{\tilde{H}}_{20} \bar{\sigma}^{\mu} \tilde{H}_{20}-\bar{\nu} \bar{\sigma}_{\nu}^{\mu}-\bar{e}_{-} \bar{\sigma}^{\mu} e_{-}+2 \bar{e}_{+} \bar{\sigma}^{\mu} e_{+}\right) \\
& +\left(e / \sin \theta_{\mathrm{w}}\right) W_{\mu}^{+}\left\{\sqrt{\frac{1}{2}}\left(\overline{\tilde{H}}_{1+} \bar{\sigma}^{\mu} \tilde{H}_{10}+\overline{\tilde{H}}_{20} \bar{\sigma}^{\mu} \tilde{H}_{2-}\right)\right. \\
& \left.+\left(-\bar{W}_{+} \bar{\sigma}^{\mu} \tilde{W}_{0}+\bar{W}_{0} \bar{\sigma}^{\mu} \tilde{W}_{-}\right)+\sqrt{\frac{1}{2}} \overline{\boldsymbol{\sigma}}^{\mu} e_{-}\right\}+ \text {h.c. } \\
& +\left(e / \sin \theta_{\mathrm{w}}\right) W_{\mu}^{0}\left\{\frac{1}{2}\left(\overline{\tilde{H}}_{1+} \bar{\sigma}^{\mu} \tilde{H}_{1+}-\overline{\tilde{H}}_{10} \bar{\sigma}^{\mu} \tilde{H}_{10}+\overline{\tilde{H}}_{20} \bar{\sigma}^{\mu} \tilde{H}_{20}-\overline{\tilde{H}}_{2-} \bar{\sigma}^{\mu} \tilde{H}_{2-}\right)\right. \\
& \left.+\left(\overline{\tilde{W}}_{+} \bar{\sigma}^{\mu} \tilde{W}_{+}-\tilde{\tilde{W}}_{-} \bar{\sigma}^{\mu} \tilde{W}_{-}\right)+\frac{1}{2}\left(\bar{\nu} \bar{\sigma}^{\mu} \nu-\bar{e}_{-} \bar{\sigma}^{\mu} e_{-}\right)\right\} .
\end{aligned}
$$

Gaugino couplings:

$$
\begin{aligned}
\mathscr{L}_{\tilde{\mathrm{G}}}= & \left(e / \cos \theta_{\mathrm{w}}\right) \frac{1}{2} \sqrt{2} i\left\{H_{1+}^{*} \tilde{B}_{0} \tilde{H}_{1+}+H_{10}^{*} \tilde{B}_{0} \tilde{H}_{10}\right. \\
& \left.-H_{20}^{*} \tilde{B}_{0} \tilde{H}_{20}-H_{2-}^{*} \tilde{B}_{0} \tilde{H}_{2-}-\tilde{\nu}^{*} \tilde{B}_{0} \nu-\tilde{e}_{-}^{*} \tilde{B}_{0} e_{-}+2 \tilde{e}_{+}^{*} \tilde{B}_{0} e_{+}\right\}+ \text {h.c. } \\
& +\left(e / \sin \theta_{\mathrm{w}}\right) i\left\{H_{1+}^{*} \tilde{W}_{+} \tilde{H}_{10}+H_{10}^{*} \tilde{W}_{-} \tilde{H}_{1+}+H_{20}^{*} \tilde{W}_{+} \tilde{H}_{2-}\right. \\
& \left.+H_{2-}^{*} \tilde{W}_{-} \tilde{H}_{20}+\tilde{\nu}^{*} \tilde{W}_{+} e_{-}+\tilde{e}_{-}^{*} \tilde{W}_{-} \nu\right\}+ \text { h.c. } \\
& +\left(e / \sin \theta_{\mathrm{w}}\right) \sqrt{\frac{1}{2}} i\left\{H_{1}^{*}, \tilde{W}_{0} \tilde{H}_{1+}-H_{10}^{*} \tilde{W}_{0} \tilde{H}_{10}+H_{20}^{*} \tilde{W}_{0} \tilde{H}_{20}\right. \\
& \left.-H_{2-}^{*} \tilde{W}_{0} \tilde{H}_{2}+\tilde{\nu}^{*} \tilde{W}_{0} \nu-\tilde{e}_{-}^{*} \tilde{W}_{0} e_{-}\right\}+ \text {h.c. }
\end{aligned}
$$


Yukawa couplings: in as far as the fermions are concerned, these are of the usual form, including the couplings of a possible singlet $Y$, all in 2-component notation:

$$
\begin{aligned}
\mathscr{L}_{Y}= & \lambda Y H_{1 i} H_{2 j} \varepsilon^{i j}+\sigma Y \tilde{Y} \tilde{Y}+\lambda_{Y}\left[Y\left(\tilde{H}_{1+} \tilde{H}_{2-}-\tilde{H}_{10} \tilde{H}_{20}\right)\right. \\
& \left.+H_{1+} \tilde{Y} \tilde{H}_{2-}-H_{10} \tilde{Y} \tilde{H}_{20}+H_{2-} \tilde{Y} \tilde{H}_{1+}-H_{20} \tilde{Y} \tilde{H}_{10}\right] \\
& +\lambda_{e}\left[e_{+} H_{20} e_{-}+\tilde{e}_{+} \tilde{H}_{20} e_{-}+e_{+} H_{20} \tilde{e}_{-}\right. \\
& \left.-e_{+} H_{2-} \nu_{e}-\tilde{e}_{+} \tilde{H}_{2-} \nu_{\mathrm{e}}-e_{+} \tilde{H}_{2-} \tilde{\nu}_{\mathrm{e}}\right]+ \text { h.c. } \\
& + \text { similar terms for the quarks. }
\end{aligned}
$$

Their scalar counterparts in the lagrangian are obtained in the standard way.

Coming to discuss the mass terms, we now have to consider in principle a huge number of different models. Let us mention first that one part of the fermion mass matrices is actually almost determined. This is the part associated with the spontaneous breaking of $\mathrm{SU}(2) \times \mathrm{U}(1)$. This is entirely described in terms of $v_{1} / \sqrt{2}=$ $\left\langle H_{10}\right\rangle$ and $v_{2} / \sqrt{2}=\left\langle H_{20}\right\rangle$, the vacuum expectation values of the two scalars $H_{1}$ and $H_{2}$. The constraint $\frac{1}{4}\left(v_{1}^{2}+v_{2}^{2}\right) g^{2}=M_{\mathrm{W}}^{2}$ leaves $v_{1} / v_{2}$ as the only parameter in this sector. The other matrix elements depend both on the mechanisms of supersymmetry breaking and on the type of model considered (e.g., the very existence of the $Y$ field). We do not want to insist here on the various mechanisms which have been proposed to break supersymmetry. The most expedient is probably to consider explicit (soft) breaking terms. This has the advantage, for our purpose, of not being restrictive. Interestingly enough, a strong motivation for introducing such a priori ugly terms in an effective lagrangian is provided by the consideration of a low-energy reduction of $N=1$ supergravity coupled to matter [7].

These models have indeed been shown to provide, at the tree level of weak interactions, "universal" masses for all the scalar fields, and R-symmetry violating trilinear scalar couplings (whose explicit form is dictated by that of the effective potential). While those scalar effective potential terms are of no immediate concern to us, they are necessary to determine the $S U(2) \times U(1)$ breaking pattern. They also imply, in higher orders in the weak coupling, the presence of "gaugino" mass terms, $\mu_{i j}$ in our matrix [8]. The value of those parameters varies considerably from model to model; actually logarithmic divergences may force the introduction of either an explicit cut-off at the Planck mass, or of explicit counterterms, therefore ruining any predictive power. We will consider various possibilities for those mass terms, essentially distinguishing between two situations: $\mathrm{SU}(2) \times \mathrm{U}(1)$ symmetrical or not.

A last word concerns the introduction of the $Y$ field. In the situation described above (scalar masses provided by supergravity) it is very tempting to completely avoid any (low-energy) explicit mass term in the lagrangian (i.e. in terms of superfields, terms like $m_{12} H_{1} H_{2}$ for instance). This, however, introduces the peril 
of a light axion, independent rotations being then allowed on the $H_{1}$ and $H_{2}$ fields. An easy way to avoid this is to introduce a singlet field $Y$. If we include both $\lambda_{Y} Y H_{1} H_{2}$ and $\frac{1}{3} \sigma Y^{3}$ terms in the lagrangian, the "overall" Peccei-Quinn symmetry is explicitly broken, (and any R-symmetry is destroyed by supergravity induced terms).

Alternatively, one may consider cases where an effective $m_{12}$ coupling will be generated by contributions emerging from symmetry breaking at the grand unification scale, for instance. In order to keep some generality, we have systematically kept the $Y$ field in our equations. In case an $m_{12}$ term is wanted instead, suffice it to replace $\lambda_{Y} Y$ by $m_{12}$ and to cancel the $Y$ entry in the neutral mass matrix.

We thus parameterize the mass matrices in terms of "known" quantities such as $v_{1}, v_{2}$ and unknowns representing the effects of higher scales.

In this 2-component notation, we get for the electron mass, from eq. (10)

$$
\frac{1}{2}\left(\begin{array}{cc}
e_{-\mathrm{L}} & e_{+\mathrm{L}} \\
0 & \lambda_{\mathrm{e}}\left\langle H_{20}\right\rangle \\
\lambda_{\mathrm{e}}\left\langle H_{20}\right\rangle & 0
\end{array}\right)^{e_{-\mathrm{L}}} \begin{gathered}
e_{+\mathrm{L}} \\
\end{gathered}
$$

which, according to the discussion above, can be rewritten as

$$
\sqrt{\frac{1}{2}} \lambda_{e} v_{2} \bar{e} e, \quad \text { where } \quad e=\left(\begin{array}{l}
e_{-\mathrm{L}} \\
\bar{e}_{+\mathrm{L}}
\end{array}\right) \text {. }
$$

In a similar way, the charged higgino-wino matrix reads:

$$
\frac{1}{2}\left(\begin{array}{cccc}
\tilde{H}_{1+} & \tilde{H}_{2-} & i \tilde{W}_{+} & i \tilde{W}_{-} \\
0 & \lambda_{Y} y & 0 & \sqrt{\frac{1}{2}} g v_{1} \\
\lambda_{Y} y & 0 & \sqrt{\frac{1}{2}} g v_{2} & 0 \\
0 & \sqrt{\frac{1}{2}} g v_{2} & 0 & \mu_{+} \\
\sqrt{\frac{1}{2}} g v_{1} & 0 & \mu_{+-} & 0
\end{array}\right) \begin{aligned}
& \tilde{H}_{1+} \\
& \tilde{H}_{2-}+\text { h.c. } \\
& i \tilde{W}_{+}
\end{aligned}
$$

(assuming charge conservation) where we have included the contributions for the $Y$ coupling, and the $\mu_{+-}$term, which represents possible explicit masses for the winos. Charge conservation allows us to use a more compact notation:

$$
\begin{aligned}
& \tilde{H}_{1+} \quad i \tilde{W}_{+} \\
& \left(\begin{array}{cc}
\lambda_{Y} y & \sqrt{\frac{1}{2}} g v_{2} \\
\sqrt{\frac{1}{2}} g v_{1} & \mu_{+-}
\end{array}\right)_{i \tilde{W}_{-}}^{\tilde{H}_{2--}}+\begin{array}{c}
\tilde{H} \\
\tilde{H}
\end{array}
\end{aligned}
$$


Similarly, for the neutrals, we have the symmetric matrix

Neutral:

\begin{tabular}{|c|c|c|c|c|}
\hline$\tilde{Y}$ & $\tilde{H}_{10}$ & $\tilde{H}_{20}$ & $i \tilde{W}_{0}$ & $i \tilde{B}$ \\
\hline$\sigma \sigma y$ & $-\sqrt{\frac{1}{2}} \lambda_{y} v_{2}$ & $-\sqrt{\frac{1}{2}} \lambda_{y} v_{1}$ & 0 & 0 \\
\hline & 0 & $-\lambda_{y} y$ & $-\frac{1}{2} g v_{1}$ & $\frac{1}{2} g^{\prime} v_{1}$ \\
\hline & & 0 & $+\frac{1}{2} g v_{2}$ & $-\frac{1}{2} g^{\prime} v_{2}$ \\
\hline & & & $\mu_{00}$ & $\mu_{0 \mathrm{~B}}$ \\
\hline
\end{tabular}

As an example and a guide in our analysis we will start from a somewhat simplistic approximation, where gaugino self-masses $\mu_{i j}$ and the field $Y$ (including its coupling to the $H$ fields) are neglected. This example actually happens to be close to some realistic models.

We denote by $w_{1}$ and $w_{2}$ the two charged Dirac particles which emerge in this case; in Weyl basis, we have

$$
\tilde{w}_{1}=\left(\begin{array}{c}
\tilde{H}_{1+} \\
-i \tilde{\tilde{W}}_{-}
\end{array}\right), \quad \tilde{w}_{2}=\left(\begin{array}{c}
\tilde{H}_{2-} \\
-i \tilde{\tilde{W}}_{+}
\end{array}\right)
$$

The mass term then reads from eq. (10),

$$
\sqrt{\frac{1}{2}} g v_{1} \overline{\tilde{w}}_{1} \tilde{w}_{1}-\sqrt{\frac{1}{2}} g v_{2} \overline{\tilde{w}}_{2} \tilde{w}_{2} .
$$

For the neutral fermions, we introduce the following combinations:

$$
\begin{aligned}
\tilde{\gamma} & =i\left(\tilde{W}_{0} \sin \theta_{\mathbf{w}}+\tilde{B}_{0} \cos \theta_{\mathbf{w}}\right), \quad(2 \text { component }), \\
\tilde{h} & \left.=\frac{v_{2}}{v} \tilde{H}_{10}+\frac{v_{1}}{v} \tilde{H}_{20}, \quad \text { (2 component }\right), \\
\tilde{w}_{0} & =\left\{\begin{array}{l}
\frac{v_{1}}{v} \tilde{H}_{10}-\frac{v_{2}}{v} \tilde{H}_{20} \\
(-i)\left(-\tilde{\bar{W}}_{0} \cos \theta+\overline{\tilde{B}}_{0} \sin \theta\right),
\end{array} \quad\right. \text { (Dirac), }
\end{aligned}
$$

while the field $\tilde{Y}$ decouples in this approximation. The mass of the Dirac $w_{0}$ is found to be

$$
m_{\tilde{\mathrm{W}}_{\mathrm{u}}}=\frac{g v}{2 \cos \theta_{\mathrm{w}}},
$$

with $v=\sqrt{v_{1}^{2}+v_{2}^{2}}$ and $m_{\mathrm{W}^{ \pm}}=\frac{1}{2} g v, m_{\mathrm{Z}}=g v / 2 \cos \theta_{\mathrm{w}}$. At this stage $\tilde{h}$ and $\tilde{\gamma}$ are still massless. Of course the terms we have neglected so far will mix those eigenstates, and provide masses to $\tilde{h}$ and $\tilde{\gamma}$. For this example, we will still write down the interaction lagrangian in terms of the eigenstates (18). 
In order to standardize the notation, we write the two 2-component spinors $\tilde{\gamma}$ and $\tilde{h}$ as Majorana four-component spinors: (distinguished by the index 0 )

$$
\tilde{\gamma}_{0} \equiv\left(\frac{\tilde{\gamma}}{\tilde{\gamma}}\right), \quad \tilde{h}_{0} \equiv\left(\frac{\tilde{h}}{\tilde{h}}\right) .
$$

Gauge couplings: $\left(\Delta=\left(v_{1}^{2}-v_{2}^{2}\right) / v^{2}, \Delta^{\prime}=v_{1} v_{2} / v^{2} ; L, R=\frac{1}{2}\left(1 \mp \gamma_{5}\right)\right)$

$$
\begin{aligned}
\mathscr{L}= & \left(e / \cos \theta_{\mathrm{w}}\right) \frac{1}{2} B_{\mu}\left[\overline{\tilde{w}}_{1} \gamma^{\mu} L \tilde{w}_{1}+\Delta \overline{\tilde{w}}_{0} \gamma^{\mu} L w_{0}+2 \Delta^{\prime} \overline{\tilde{w}}_{0} \gamma^{\mu} L \tilde{h}_{0}\right. \\
& \left.-\overline{\tilde{w}}_{2} \gamma^{\mu} L \tilde{w}_{2}-\Delta \overline{\tilde{h}}_{0} \gamma^{\mu} L \tilde{h}_{0}+2 \Delta^{\prime} \tilde{h}_{0} \gamma^{\mu} L \tilde{w}_{0}\right] \\
& +\left(e / \sin \theta_{\mathrm{w}}\right) W_{\mu}^{+}\left\{\sqrt { \frac { 1 } { 2 } } \left[\frac{v_{1}}{v} \overline{\tilde{w}}_{1} \gamma^{\mu} L \tilde{w}_{0}+\frac{v_{2}}{v} \overline{\tilde{w}}_{1} \gamma^{\mu} L \tilde{h}_{0}\right.\right. \\
& \left.+\frac{v_{1}}{v} \overline{\tilde{h}}_{0} \gamma^{\mu} L \tilde{w}_{2}-\frac{v_{2}}{v} \overline{\tilde{w}}_{0} \gamma^{\mu} L \tilde{w}_{2}\right] \\
& +\sin \theta_{\mathrm{w}} \overline{\tilde{\gamma}}_{0} \gamma^{\mu} R \tilde{w}_{2}-\sin \theta_{\mathrm{w}} \overline{\tilde{w}}_{1} \gamma^{\mu} R \tilde{\gamma}_{0} \\
& \left.-\cos \theta_{\mathrm{w}} \overline{\tilde{w}}_{0} \gamma^{\mu} R \tilde{w}_{2}+\cos \theta_{\mathrm{w}} \tilde{\tilde{w}}_{1} \gamma^{\mu} R \tilde{w}_{0}\right\}+ \text { h.c. } \\
& +\left(e / \sin \theta_{\mathrm{w}}\right) W_{0 \mu}\left[\frac { 1 } { 2 } \left(\overline{\tilde{w}}_{1} \gamma^{\mu} L \tilde{w}_{1}-\overline{\tilde{w}}_{2} \gamma^{\mu} L \tilde{w}_{2}-\Delta \overline{\tilde{w}}_{0} \gamma^{\mu} L \tilde{w}_{0}-2 \Delta^{\prime} \overline{\tilde{w}}_{0} \gamma^{\mu} L \tilde{h}_{0}\right.\right. \\
& \left.\left.+\Delta \tilde{h}_{0} \gamma^{\mu} L \tilde{h}_{0}-2 \Delta^{\prime} \overline{\tilde{h}}_{0} \gamma^{\mu} L \tilde{w}_{0}\right)-\overline{\tilde{w}}_{2} \gamma^{\mu} R \tilde{w}_{2}+\overline{\tilde{w}}_{1} \gamma^{\mu} R \tilde{w}_{1}\right] \\
& +\operatorname{standard~terms~for~leptons~and~quarks.~}
\end{aligned}
$$

\section{Gaugino couplings :}

$$
\begin{aligned}
& +\left(e / \sin \theta_{\mathrm{w}}\right)\left[\frac{v_{1}}{v} H_{1+}^{*} \overline{\tilde{w}}_{2 \mathrm{R}} \tilde{w}_{0 \mathrm{~L}}+H_{10}^{*} \overline{\tilde{w}}_{1 \mathrm{R}} \tilde{w}_{1 \mathrm{~L}}+\frac{v_{2}}{v} H_{1+}^{*} \overline{\tilde{w}}_{2 \mathrm{R}} \tilde{h}_{0 \mathrm{~L}}+H_{20}^{*} \overline{\tilde{w}}_{2 \mathrm{R}} \tilde{w}_{2 \mathrm{~L}}\right. \\
& \left.+\frac{v_{1}}{v} H_{2-}^{*} \overline{\tilde{w}}_{1 \mathrm{R}} \tilde{h}_{0 \mathrm{~L}}-\frac{v_{2}}{v} H_{2-}^{*} \overline{\tilde{w}}_{1 \mathrm{R}} \tilde{w}_{\mathrm{OL}}+\tilde{\nu}^{*} \overline{\tilde{w}}_{2} e_{-\mathrm{L}}+\tilde{e}_{-}^{*} \overline{\tilde{w}}_{1} \nu_{\mathrm{L}}\right] \\
& +\sqrt{\frac{\mathrm{T}}{2}}\left(e / \sin \theta_{\mathrm{w}}\right)\left(-\overline{\tilde{w}}_{0 \mathrm{R}} \cos \theta_{\mathrm{w}}+\overline{\tilde{\gamma}}_{0 \mathrm{R}} \sin \theta_{\mathrm{w}}\right) \\
& \times\left(H_{1+}^{*} \tilde{w}_{1 \mathrm{~L}}-H_{2-}^{*} \tilde{w}_{2 \mathrm{~L}}-H_{10}^{*} \frac{v_{1}}{v} \tilde{w}_{0 \mathrm{~L}}-H_{10}^{*} \frac{v_{2}}{v} \tilde{h}_{\mathrm{OL}}\right. \\
& \left.+H_{20}^{*} \frac{v_{1}}{v} \tilde{h}_{\mathrm{OL}}-H_{20}^{*} \frac{v_{1}}{v} \tilde{w}_{\mathrm{OL}}+\tilde{\nu}^{*} \nu_{\mathrm{L}}-\tilde{e}_{-}^{*} e_{\mathrm{L}}\right) \\
& +\sqrt{\frac{1}{2}}\left(e / \cos \theta_{\mathrm{w}}\right)\left(\overline{\tilde{w}}_{O \mathrm{R}} \sin \theta_{\mathrm{w}}+\overline{\tilde{\gamma}}_{\mathrm{OR}} \cos \theta_{\mathrm{w}}\right) \\
& \times\left(H_{1+}^{*} \tilde{w}_{1 \mathrm{~L}}-H_{2-}^{*} \tilde{w}_{2 \mathrm{~L}}+H_{10}^{*} \frac{v_{1}}{v} \tilde{w}_{\mathrm{OL}}+H_{10}^{*} \frac{v_{2}}{v} \tilde{h}_{\mathrm{OL}}-H_{20}^{*} \frac{v_{1}}{v} \tilde{h}_{\mathrm{OL}}\right. \\
& \left.+H_{20}^{*} \frac{v_{2}}{v} \tilde{w}_{\mathrm{OL}}-\tilde{e}^{*} e_{\mathrm{L}}-\tilde{\nu}^{*} \nu_{\mathrm{L}}+2 \tilde{e}_{+e}^{*} e_{+\mathrm{L}}\right)+ \text { h.c. }{ }^{\star}
\end{aligned}
$$

* We have omitted in (20) similar term arising from the Yukawa coupling (10). This is justified for the leptons, since $\lambda_{c} \ll e$. 
From this lagrangian or eqs. (9) and (18), we can read off all the couplings we will need in the following sections.

Finally, at this stage there are two massless neutral fermions, $\tilde{h}_{0}$ and $\tilde{\gamma}_{0}$. Generally they will mix and separate, as well as both getting some mass. After further diagonalization one will have a lightest state, which we call $\tilde{\gamma}_{1}$ and refer to as the photino, and a second lightest state $\tilde{\gamma}_{2}$. We discuss their properties in sect. 4 .

In order to get some insight into the type of particles we are actually hunting for in more realistic situations, we will consider below some numerical values, corresponding to a sample of "typical" cases.

As far as charged states are concerned, sect. 4 considers two extreme cases, where either off-diagonal entries $\left(g v_{i}\right)$ or diagonal ones dominate. This latter case was considered in detail by Ellis and Ross, ref. [2]. We have Dirac pairs in all cases. The diagonalization of the $5 \times 5$ neutral mass matrix proves more complicated, and in general we have to stick to Majorana states. We distinguish between two situations

$$
\text { (i) }\left|v_{1} / v_{2}\right|=1, \quad \text { (ii) } \quad\left|v_{1} / v_{2}\right| \neq 1 \text {. }
$$

The first case is met in a class of models where supersymmetry breaking in a "hidden sector" induces soft supersymmetry breaking terms in the low-energy effective $S U(2) \times U(1)$ lagrangian (no grand unification implied) [7]. Since these terms are "universal" at the tree level, $H_{1}$ and $H_{2}$ are treated symmetrically (apart from Yukawa couplings to ordinary fermions) and a local minimum with $v_{1}=v_{2}$ exists under certain conditions.

Gaugino masses arise at the one-loop level (if not introduced explicitly) and are dominated by the t-quark (+partner) contribution. (This implies $\left|\mu_{\mathrm{WB}} / \mu_{\mathrm{BB}}\right|=\frac{3}{2}$.)

Assuming the values (in $\mathrm{GeV}$ )

$$
\begin{gathered}
\sigma y=80, \quad \sqrt{\frac{1}{2}} \lambda v_{1}=30, \quad \sqrt{\frac{1}{2}} \sigma v_{1}=11.25, \quad \sqrt{\frac{1}{2}} g v_{1}=57, \quad g^{\prime} / g=0.5, \\
\mu_{\mathrm{BB}}=2, \quad \mu_{\mathrm{WB}_{\mathrm{B}}}=3 .
\end{gathered}
$$

We find the lower lying eigenstates to be: (note that for Majorana particles the lagrangian mass term reads $\frac{1}{2} m \bar{\psi} \psi$ with $m$ the mass)

\begin{tabular}{lcccccc} 
& Mass & \multicolumn{5}{c}{ Components } \\
$\tilde{\gamma}_{1}$ & 0.79 & - & $\tilde{H}_{10}$ & $\tilde{H}_{20}$ & $i \tilde{W}_{0}$ & $i \tilde{B}_{0}$ \\
$\tilde{\gamma}_{2}$ & 28 & 0.36 & 0.02 & -0.02 & -0.44 & -0.89 \\
$\tilde{\gamma}_{3}$ & 82.6 & - & -0.49 & 0.66 & - & - \\
& & - & 0.49 & -0.66 & 0.30.
\end{tabular}

Note that in this case the appelation $\tilde{\gamma}_{2}$ is badly deserved, since this state has negligible gaugino components!

Other values of $\lambda$ and $\sigma$ barely alter this conclusion. (Note that the $\mathrm{SU}(2) \times \mathrm{U}(1)$ symmetry breaking $\mu_{\mathrm{WB}}, \mu_{\mathrm{BB}}$ should remain small in this case.) For instance, if we 
omit the $Y$ field and provide an explicit mass for $H_{1}, H_{2}$,

$\begin{array}{cccccc} & m_{12}=30, & \sqrt{\frac{1}{2}} g v_{1}=57, & \mu_{\mathrm{BB}}=2, & \mu_{\mathrm{WB}}=3, \\ & \text { Mass } & \tilde{H}_{10} & \tilde{H}_{20} & i \tilde{W}_{0} & i \tilde{B}_{0} \\ \tilde{\gamma}_{1} & 0.77 & 2 \cdot 10^{-2} & 2 \cdot 10^{-2} & -0.44 & -0.9 \\ \tilde{\gamma}_{2} & 30 & \sqrt{ } 2 & \sqrt{ } 2 & - & - \\ \tilde{\gamma}_{3} & 74 & -0.46 & 0.46 & -0.69 & 0.31 .\end{array}$

As a result, the " $\tilde{\gamma}_{2}$ " is very weakly coupled to ordinary particles in this case (only via Yukawa couplings) and will be difficult to produce.

The conspicuous signature of a $\tilde{\gamma}_{1} \tilde{\gamma}_{2}$ production cross section in sect. 4 would thus await higher available energies, (SLC, LEP) where $\tilde{\gamma}_{3}$ can take the place of an elusive $\tilde{\gamma}_{2}$. We should however mention paranthetically that these models are at present in considerable jeopardy due to the existence of other, shallower minima. Extra (heavy) particles are to be included to stabilize the above mentioned minimum, and this makes the constriction very unattractive. (Alternatively, a degeneracy of the vacuum might be invoked, see ref. [7].)

In other models, the previous scheme of supersymmetry breaking is adhered to initially, but the renormalization group evolution of the induced mass terms is followed down from the "grand unification" scale $[5,6]$. this may lead to a negative mass term for the $H_{1}$ boson. Usually, no $Y$ field is assumed and the quartic part of the potential is provided by the $D$ terms. Typically in these terms $v_{1} \gg v_{2}$.

We list some numerical values for models with $v_{1} \neq v_{2}$ assuming an explicit (or induced) $m_{12}$ term,

\begin{tabular}{|c|c|c|c|c|c|}
\hline \multirow[t]{2}{*}{$v_{1} / v_{2}=10$} & \multicolumn{2}{|c|}{$m_{12}=10 \mathrm{GeV}$} & \multicolumn{2}{|c|}{$\mu_{\mathrm{ww}}=80 \mathrm{geV}$} & $\mu_{\mathrm{BB}}=20 \mathrm{GeV}$ \\
\hline & Mass & $\tilde{H}_{10}$ & $\tilde{H}_{20}$ & $i \tilde{W}$ & $i \tilde{B}$ \\
\hline$\tilde{\gamma}_{1}$ & 2.51 & 0.15 & 0.98 & +0.06 & -0.12 \\
\hline$\tilde{\gamma}_{2}$ & 30.2 & 0.23 & 0.06 & +0.37 & 0.9 \\
\hline$\tilde{\gamma}_{3}$ & 68.3 & 0.79 & -0.20 & +0.44 & -0.38 \\
\hline \multicolumn{2}{|c|}{$v_{1} / v_{2}=10$} & $m_{12}=10$ & \multicolumn{2}{|c|}{$\mu_{\mathrm{wW}}=30$} & $\mu_{\mathrm{BB}}=20$ \\
\hline & Mass & $\tilde{H}_{10}$ & $\tilde{H}_{20}$ & $i \tilde{W}$ & $i \tilde{B}$ \\
\hline$\tilde{\gamma}_{1}$ & 2.28 & 0.13 & 0.99 & +0.08 & -0.07 \\
\hline$\tilde{\gamma}_{2}$ & 22.0 & 0.04 & 0.02 & +0.44 & 0.9 \\
\hline \multirow[t]{3}{*}{$\tilde{\gamma}_{3}$} & 78.9 & 0.75 & -0.17 & +0.56 & -0.31 \\
\hline & $2=3$ & $m_{12}=10$ & $\mu_{\mathrm{wW}}$ & $\mu_{\mathrm{B}}$ & 20 \\
\hline & Mass & $\tilde{H}_{10}$ & $\tilde{H}_{20}$ & $i \tilde{W}$ & $i \tilde{B}$ \\
\hline$\tilde{\gamma}_{1}$ & 6.24 & 0.35 & 0.93 & +0.04 & -0.1 \\
\hline$\tilde{\gamma}_{2}$ & 30.0 & 0.24 & - & +0.36 & 0.90 \\
\hline$\tilde{\gamma}_{3}$ & 68.0 & 0.75 & -0.34 & +0.44 & -0.37 \\
\hline
\end{tabular}


For this class of models one finds a detectable $\mathrm{e}^{+} \mathrm{e}^{-} \rightarrow \tilde{\gamma}_{2} \tilde{\gamma}_{1}$ cross section even at present energies (see sect. 4).

\section{Charged fermion-Higgs and gauginos}

In the following, we will denote the lightest, charged mass eigenstate as $\tilde{h}^{+}$, keeping in mind that its couplings may be quite different from those for a fermionHiggs because of mixing with winos. Similarly, $\tilde{\gamma}_{1}, \tilde{\gamma}_{2}$ will be the light neutral states, as described in tables 1-3. In terms of sect. $2, \tilde{h}^{+}$can be $\tilde{w}_{1}$ or $\tilde{w}_{2}$.

First we discuss the production of $\tilde{h}^{+}$, including the forward-backward asymmetry in $\mathrm{e}^{+} \mathrm{e}^{-}$reactions, and then the decay signatures. In the decay section we explain why $\tilde{h}^{ \pm}$might not have been detected in present data even if it were there, as well as how to proceed with future data.

\subsection{PRODUCTION}

3.1.1 $e^{+} e^{-}$. Since $\tilde{h}^{+}$is a charged, spin- $-\frac{1}{2}$ object it will be produced with one unit of $R$ via the photon in $\mathrm{e}^{+} \mathrm{e}^{-}$interactions. There is a further contribution from the $Z^{0}$. This means thousands of events of $\tilde{h}^{+} \tilde{h}^{-}$would have been produced at PETRA/PEP if $\tilde{h}^{+}$were not too heavy, which has led everyone to assume that $m\left(h^{+}\right) \geqslant 18 \mathrm{GeV}$. While that conclusion might be correct, we will show below that the decay characteristics are sufficiently subtle that $\tilde{h}^{+}$might still be found with lighter masses.

The production in $\mathrm{e}^{+} \mathrm{e}^{-}$is standard, with 1 unit of $R$, a $1+\cos ^{2} \theta$ angular distribution (apart from $\mathrm{Z}^{0}$ effects), and a threshold factor $\beta=\sqrt{1-4 \pi^{2} / s}$, all just as for a new charged lepton.

If a new state were found, further measurements of the forward-backward asymmetry and the absolute cross section would be needed to distinguish it from a conventional sequential lepton (this has already been mentioned by Ellis and Ross [2]). Table 4 gives the vector and axial vector couplings that arise for various (perhaps likely) limiting cases; any intermediate answer is possible.

TABLE 4

\begin{tabular}{lccc}
\hline Mass eigenstate & $g_{\mathrm{v}}$ & $g_{\mathrm{a}}$ & $g_{\mathrm{v}}^{2}+\mathrm{g}_{\mathrm{a}}^{2}$ \\
\hline (a) normal lepton $\ell^{-}$ & $-\frac{1}{2}+2 \sin ^{2} \theta_{\mathrm{w}}$ & $\frac{1}{2}$ & 0.26 \\
(b) $\dot{H}^{-}+\tilde{H}^{+*}$ & $-1+2 \sin ^{2} \theta_{\mathrm{w}}$ & 0 & 0.31 \\
(c) $\tilde{W}^{-}+\tilde{W}^{+*}$ & $-2+2 \sin ^{2} \theta_{\mathrm{w}}$ & 0 & 2.43 \\
(d) $\tilde{W}^{-}+\tilde{H}^{+*}$ & $-\frac{3}{2}+2 \sin ^{2} \theta_{\mathrm{w}}$ & $\frac{1}{2}$ & 1.37 \\
(e) $\tilde{H}^{-}+\tilde{W}^{+*}$ & $-\frac{3}{2}+2 \sin ^{2} \theta_{\mathrm{w}}$ & $-\frac{1}{2}$ & 1.37
\end{tabular}

The neutral current couplings of various mass eigenstates are given for purely diagonal and off-diagonal mass matrices and compared to those for a normal lepton. Cases (b), (c) have no forward-backward asymmetry, (a) has that of a normal lepton, and (d), (e) have large forward-backward asymmetries of opposite sign, all shown in fig. 1 . 
To distinguish various situations one can study the differential cross section at a given energy, or the total cross section versus energy. These are (including $\gamma, Z^{0}$ contributions)

$$
\begin{aligned}
\frac{\mathrm{d} \sigma}{\mathrm{d} \Omega} & =\frac{\alpha^{2}}{4 s}\left\{(1+A)\left(1+\cos ^{2} \theta\right)+2 A^{\prime} \cos \theta\right\}, \\
A & =8 g_{\mathrm{v}} g_{\mathrm{ve}} \operatorname{Re} \chi+16\left(g_{\mathrm{v}}^{2}+g_{\mathrm{a}}^{2}\right)\left(g_{\mathrm{ve}}^{2}+g_{\mathrm{ae}}^{2}\right)|\chi|^{2}, \\
A^{\prime} & =8 g_{\mathrm{a}} g_{\mathrm{ae}} \operatorname{Re} \chi+64 g_{\mathrm{v}} g_{\mathrm{a}} g_{\mathrm{ve}} g_{\mathrm{ae}}|\chi|^{2}, \\
\chi & =\frac{G_{\mathrm{F}} s m_{\mathrm{Z}}^{2}}{8 \sqrt{2} \pi \alpha} \frac{1}{\left(s-m_{\mathrm{Z}}^{2}\right)-i m_{\mathrm{Z}} \Gamma_{\mathrm{Z}}}, \\
A_{\mathrm{FB}} & =(\mathrm{d} \sigma(\theta)-\mathrm{d} \sigma(\pi-\theta)) /(\mathrm{d} \sigma(\theta)+\mathrm{d} \sigma(\pi-\theta))=\frac{2 \cos \theta}{1+\cos ^{2} \theta} \frac{A^{\prime}}{1+A} .
\end{aligned}
$$

In fig. 1 the quantity $A^{\prime} /(1+A)$, which determines the forward-backward asymmetry is plotted for the five cases. Combining rates with the asymmetry, they can clearly be distinguished. Models of the Ellis-Ross sort, with mainly diagonal mass matrices because of a large gaugino mass contribution, give essentially vector-like fermions, and no asymmetry. Models with mainly off diagonal mass matrices give

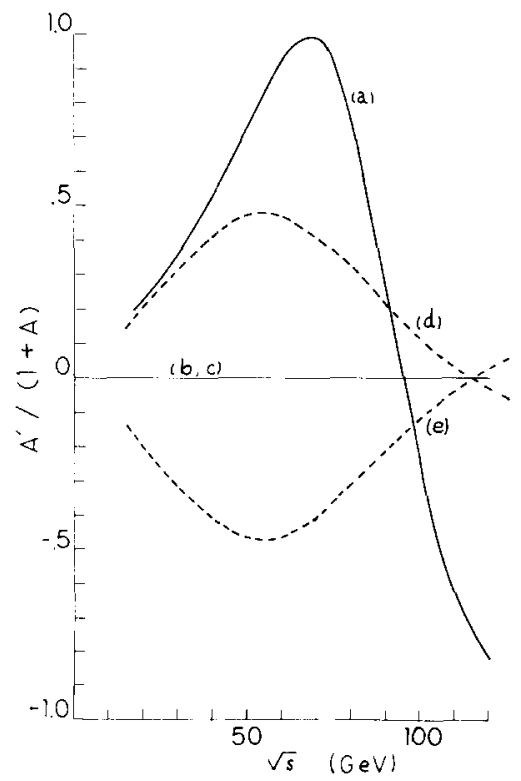

Fig. 1. Forward-backward asymmetries that allow a wino or a fermion-Higgs to be distinguished from a lepton and from each other. 
a large asymmetry. When such states are discovered it will be easy to untangle the mixing and determine the form of the mass matrix.

3.1.2. $p p, \bar{p} p$ colliders. The particles we are discussing will not be produced directly with large cross sections, but they will occur in $\mathrm{W}^{ \pm}, \mathrm{Z}^{0}$ decay, perhaps allowing them to be found there.

The $\mathrm{W}^{+} \tilde{h}^{-} \tilde{\gamma}$ coupling is e, from the lagrangian, so the expected branching ratio from $W$ decay or explicit production is about

$$
\operatorname{BR}\left(\tilde{\mathbf{W}}^{+} \tilde{\gamma}\right) / \operatorname{BR}(\mathrm{e} \nu)=2 e^{2} / g^{2}=0.41,
$$

up to further mixing angles for the removal of the degeneracy giving $\tilde{\gamma}_{1}, \tilde{\gamma}_{2}$. That is, for every event of $\mathrm{W} \rightarrow \mathrm{e} \nu$, then there will be at least $\left(\delta \equiv m_{\mathrm{h}}^{2} / m_{\mathrm{W}}^{2}\right)$

$$
0.41 \times(1-\delta)\left(1-\frac{1}{2} \delta+\frac{1}{2} \delta^{2}\right)
$$

events of $\mathrm{W}^{ \pm} \rightarrow \tilde{h}^{ \pm} \tilde{\gamma}$. This has already been emphasized by Weinberg [4], and discussed by Arnowitt et al. [3]. Depending on the $\tilde{h}^{ \pm}$decay, these may be detectable; see the discussion on signatures below. The $\tilde{\gamma}$ will escape, so the basic signature is large missing $p_{\mathrm{T}}$, along with the $\tilde{\mathrm{h}}^{ \pm}$or its decay products. Probably the best signature is the large missing $E_{\mathrm{T}}$ from the escaping photino, and no associated hard lepton. It would seem possible to at least put an upper limit on the presence of such events.

One can also [3] have $\mathrm{Z}^{0} \rightarrow \tilde{\mathrm{h}}^{+} \tilde{\mathrm{h}}^{-}, \tilde{\mathrm{w}}^{+} \tilde{\mathrm{h}}^{-}$. We will trace through the signatures below.

\subsection{DECAYS}

These are four possible ways that $\tilde{h}^{ \pm}$can behave. The first two, while they should be considered for completeness, do not at the moment occur in favored models. A useful recent review of the experimental situation is given in ref. [9].

(a) $\tilde{h}^{ \pm}$could be stable or very long-lived. Then a pair of stable particles of any kinematically allowed mass would be produced in $\mathrm{e}^{+} \mathrm{e}^{-} \rightarrow \tilde{\mathrm{h}}^{+} \tilde{\mathrm{h}}^{-}$(or $\mathrm{W}^{ \pm} \rightarrow \tilde{\mathrm{h}}^{ \pm} \tilde{\gamma}$ at a hadron collider). The MARK J [10] and JADE [11] collaborations have looked for such objects and give lower limits on the mass of $14 \mathrm{GeV}$ and $12 \mathrm{GeV}$ respectively. It would be useful to have stronger restrictions published.

(b) If there is a charged Higgs $\mathrm{H}^{ \pm}$lighter than $\tilde{\mathrm{h}}^{ \pm}$, the latter can decay via $\tilde{\mathbf{h}}^{ \pm} \rightarrow \mathrm{H}^{ \pm} \tilde{\gamma}$. Although $\mathrm{H}^{ \pm}$for some masses and branching ratios are excluded, there are windows in the relevant region e.g. for $m\left(\mathrm{H}^{ \pm}\right)=12 \mathrm{GeV}, \mathrm{BR}\left(\mathrm{H}^{ \pm} \rightarrow \tau^{ \pm} \nu_{\tau}\right)=15 \%$ (as would be expected for simple coupling proportional to masses, plus color counting). If $\tilde{h}^{ \pm} \rightarrow \mathrm{H}^{ \pm} \tilde{\gamma}$ were allowed it would be semiweak and would dominate the alternatives discussed below. In currently favored supersymmetric models $\mathbf{H}^{ \pm}$ is heavier than $\tilde{h}^{ \pm}$, but that depends on the structure of the mass matrices chosen.

(c) If (a), (b) do not ocurr, then $\tilde{h}^{ \pm}$can $\beta$-decay [2-4] to a lighter, neutral supersymmetric state, as in fig. $2 \mathrm{a}, 2 \mathrm{~b}$. Apart from possible mixing effects at the 


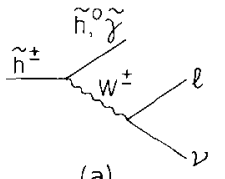

(a)

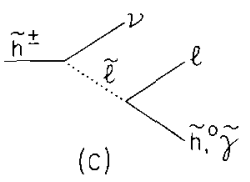

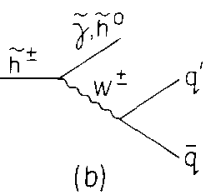

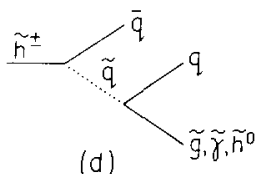

(d)

Fig. 2. Decay modes of charged fermion-Higgs.

upper vertex, this is a standard weak decay, with an associated lifetime $\sim 10^{-13}$ sec (assuming $\dot{\mathrm{H}}^{ \pm}$and the neutral supersymmetric state are separated in mass by $\geqslant 1 \mathrm{GeV}$ ). The possibility should not be ignored that such a state could show up as a long-lived particle in experiments with a vertex detector.

That the decay of fig. 2 a could occur has led people to believe that $\tilde{h}^{ \pm}$of mass below the top PETRA/PEP energies was excluded, because it closely resembles a sequential lepton, which has been looked for. However, the present case is a little different, and published experiments usually have cuts which might have reduced the signal to an undetectable level.

The reason is simply kinematical. When the neutral particle at the upper vertex has mass zero, the spectrum of the charged lepton can rise to its endpoint as it is assumed to do for sequential lepton searches, while when the neutral particle has signficant mass the spectrum is cut off. Further, to the extent the coupling at the upper vertex in fig. $2 \mathrm{a}$ is right-handed, the spectrum of the charged lepton is pushed further to the soft side. These effects are shown in fig. 3 , for the numerical example $m\left(\tilde{\mathrm{h}}^{+}\right)=10 \mathrm{GeV}, m\left(\tilde{\mathrm{h}}^{0}\right)=4 \mathrm{GeV}$. If the standard sequential case with $m_{0}=0$ and the right-handed case with $m_{0}=4 \mathrm{GeV}$ are normalized to the same total area, the fraction of events with the energy of the charged lepton above $3 \mathrm{GeV}$ is three times smaller for the latter case. Alternatively, only $23 \%$ of the events for the case with $m_{0}=4 \mathrm{GeV}$, right-handed have the charged lepton energy above $3 \mathrm{GeV}$. While the precise relevance of these numbers has to be evaluated for each detector, with kinematics treated correctly in detail, the basic effect is that a factor of order 3 in sensitivity is lost, and such events might have been missed. We are not arguing that such events were missed, but that the question of finding supersymmetric partners is too important not to carefully check all alternatives.

An alternative way to deal with the numbers is along the following lines. Initially one has $\sqrt{1-4 m_{+}^{2} / s}$ units of $R$. For $m_{+}=10 \mathrm{GeV}$ and $\sqrt{ } s=30$ this is $\frac{3}{4}$ units. Since the $\mathrm{e}+\mu$ modes are about $2 /(3+3+3+2)=\frac{2}{11}$ of the total of fig. $2 \mathrm{a}, \mathrm{b}$ one is down to 0.14 units of $R$. A further reduction of 4 by a cut on lepton energy, and (say) a factor of 0.7 for detection efficiencies gives finally 0.024 units of $R$. Since one unit of $R$ is $0.1 \mathrm{nb}, 10^{4} \mathrm{nb}^{-1}$ of integrated luminosity gives only 24 events finally 


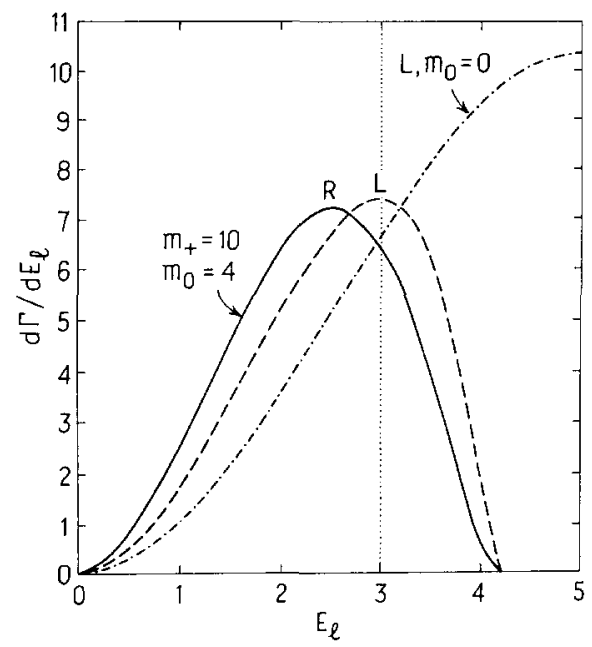

Fig. 3. Spectrum of the lepton from fig. 2a for different mass values and couplings, showing that a cut on $E_{\ell} \geqslant 3 \mathrm{GeV}$ will eliminate a large part of the signal.

detected (half are e $\mu$ events). While this may be enough, some care is necessary. Further, most remaining events have momenta near the cut-off energy. Finally, note all the above assumes no other competing decays. It could happen that fig. $2 \mathrm{~d}$ dominates and further reduces the available number of detectable charged leptons.

For completeness we give the spectrum for the decay of fig. 2a for the charged lepton in fig. 3. If there is a factor $\left(g_{2} / 2 \sqrt{ } 2\right) \overline{\bar{h}}^{0} \gamma_{\mu}\left[L P_{\mathrm{L}}+R P_{\mathrm{R}}\right] \bar{h}^{+}$at the upper vertex ( $L, R$ are couplings and $P_{\mathrm{L}}, P_{\mathrm{R}}$ projection operators) and $\left(g_{2} / 2 \sqrt{2}\right) \bar{\ell}^{-} \gamma_{\mu} P_{\mathrm{L}} \nu$ at the lower vertex, then with $E=$ the charged lepton energy, and neglecting the mass of the charged lepton,

$$
\begin{aligned}
\mathrm{d} \Gamma / \mathrm{d} E & =\left(L^{2} \Gamma_{\mathrm{L}}+R^{2} \Gamma_{\mathrm{R}}+L R \Gamma_{\mathrm{LR}}\right) m_{+} G_{\mathrm{F}}^{2} / \pi^{3}, \\
\Gamma_{\mathrm{L}} & =E^{2}\left[\left(E_{\max }-E\right) k+\frac{1}{2}\left(2 E-E_{\max }\right) k^{2}-\frac{1}{3} E k^{3}\right], \\
\Gamma_{\mathrm{R}} & =E^{2}\left[E_{\max }-E\right] k, \quad \Gamma_{\mathrm{LR}}=-\frac{1}{2} m_{0} E^{2} k^{2}, \\
k & =\left(m_{+}^{2}-m_{0}^{2}-2 m_{+} E\right) /\left(m_{+}-2 E\right) m_{+}=\left(E_{\max }-E\right) /\left(\frac{1}{2} m_{+}-E\right), \\
E_{\max } & =\left(m_{+}^{2}-m_{0}^{2}\right) / 2 M_{+} .
\end{aligned}
$$

Since $k \equiv 1$ if $m_{0}=0$ but $k$ vanishes at $E=E_{\max }$ if $m_{0} \neq 0$ one can see the origin of the qualitative change introduced by $m_{0} \neq 0$.

(d) Finally, one can have [2-4]

$$
\tilde{\mathrm{h}}^{ \pm} \rightarrow \mathrm{q}^{\prime} \overline{\mathrm{q}} \tilde{\gamma},
$$

(or $\mathrm{q} \overline{\mathrm{q}} \tilde{\mathrm{g}}, \mathrm{q} \overline{\mathrm{q}} \tilde{h}^{0}$ depending on masses). Which decay dominates depends on the 
scalar quark mass compared to the scalar lepton mass and the $\mathrm{W}$ mass, and on the form of the coupling at the upper vertex. If the decay via W's dominates, then q' $\bar{q}$ will always dominate because of color. If the $\tilde{h}^{ \pm}$couples mainly as a Higgs, proportional to fermion masses, the contributions of $2 \mathrm{c}, 2 \mathrm{~d}$ will be strongly suppressed. If $\tilde{h}^{ \pm}$couples with a significant fraction of wino, with a strength given by the gauge coupling and no mass factor, $2 \mathrm{c}$ and $2 \mathrm{~d}$ could dominate. It would be easy to arrange comparable contributions for $\mathrm{q}^{\prime} \overline{\mathrm{q}} \tilde{\gamma}$ and $\ell \nu \tilde{\gamma}$, further decreasing the number of $\ell \nu \tilde{\gamma}$ events.

Would the $\mathrm{q}^{\prime} \overline{\mathrm{q}} \tilde{\gamma}$ decays have been observed? From the point of view of events of distinct topology they are rather complicated, with four-quark jets. They do have two escaping $\tilde{\gamma}$ which can give useful $p_{\mathrm{T}}$ imbalances, but we do not know of published results which limits the occurrence of such events.

Alternatively, such events might show up as a step in $R$. For $\beta=0.75$ one begins with about $0.75 \times \frac{8}{11} \approx 0.55$ units of $R$ to search for. However, most $R$ searches have made cuts such as not including events with less than $50 \%$ of $\sqrt{ } s$ detected. Here the $\tilde{\gamma}$ 's escape and so does part of the q'a energy from missing $K_{\mathrm{L}}$, neutrons, detection inefficiencies, etc. (we gather from discussions that $15 \%$ is a typical number), so often these events will not pass the cut and will not be included in $R$.

To give a quantitative example, we assume the decay of fig. $2 \mathrm{~d}$. Then the distribution of $\tilde{\gamma}$ energies is

$$
\mathrm{d} \Gamma / \mathrm{d} x \sim \sqrt{x^{2}-m^{2} / M^{2}}\left\{x\left(1-\frac{4}{3} x\right)-\left(\frac{2}{3}-x\right) m^{2} / M^{2}\right\},
$$

where the photino mass is $m$, the $\tilde{\mathrm{h}}^{ \pm}$mass is $M$, and quark masses are ignored. The variable $x$ is defined by

$$
x=E_{\tilde{\gamma}} / M
$$

with $x_{\min }=m / M$ and $x_{\max }=\frac{1}{2}$. If we take $M=12 \mathrm{GeV}, m=3 \mathrm{GeV}$ we find that the mean $x$ is 0.42 , while for $m=0$ the mean $x$ is 0.37 since the distribution is still peaked at large $x$, so we assume $x=0.40$ as a relevant example. Then for (say) $M=12 \mathrm{GeV}, m=2 \mathrm{GeV}$ about $8 \mathrm{GeV}$ of energy is left for $\mathrm{q}^{\prime} \overline{\mathrm{q}}$ on each side. If $15 \%$ of this escapes a typical event has only $45 \%$ of the input energy left and would not survive an $R$ cut. Of course, for real events there is a distribution and it is a quantitative question depending on the detectors and the cuts whether such events could have escaped detection. It seems likely that an absolute $R$ measurement would not find these events. However, a relative search may be able to rule out such a step. Whether it could be excluded depends sufficiently sensitively on what happens with the cuts and the events with $E \simeq \frac{1}{2} \sqrt{ } s$ that we are unable to judge from published information we have found.

To summarize this section: each of the ways a charged supersymmetric fermionHiggs decays might differ sufficiently from the decay of a standard particle to cause such a fermion to have escaped detection so far at $\mathrm{e}^{+} \mathrm{e}^{-}$colliders. By looking at data with such a situation in mind and loosening cuts somewhat, it will be possible 
to exclude such particles or find them if they exist. Analyses at future machines should consider carefully what cuts are being made, and vary them where possible.

\section{Neutral fermion-Higgs and gauginos}

As discussed in sect. 2, there should be (at least) four neutral, spin $-\frac{1}{2}$ supersymmetric partners. The mass eigenstates may be complicated combinations of the weak eigenstates, so the coupling patterns have to be kept track of carefully. In this section we concentrate on the possibilities for detecting the neutral fermionHiggs at existing $\mathrm{e}^{+} \mathrm{e}^{-}$colliders CESR, PEP, PETRA. The analysis will directly apply at future machines as well.

The most interesting possibility may be the following. The two lightest states, $\tilde{\gamma}_{1}$ and $\tilde{\gamma}_{2}$, are produced by scalar-electron exchange as in fig. $4 \mathrm{a}$. The cross section is typically weak; we estimate it below. It should be emphasized that if $\tilde{\gamma}_{1}$ is quite light, this process is sensitive for $m\left(\tilde{\gamma}_{2}\right)$ nearly up to $\sqrt{ } s$. Then $\tilde{\gamma}_{2}$ decays as in fig. $4 \mathrm{~b}$. The diagram with quarks in the decay, counting the two lowest families and color, gives most of the final states, with a branching ratio of about $\frac{1}{5}$ for $\mathrm{e}^{+} \mathrm{e}^{-}$and $\frac{1}{5}$ for $\mu^{+} \mu^{-}$. The $\tilde{\gamma}_{1}$ escapes the detector. Thus there is a class of events with no energy detected in one hemisphere, and $\mathrm{e}^{+} \mathrm{e}^{-}$or $\mu^{+} \mu^{-}$or $\mathrm{q} \overline{\mathrm{q}}$ (large multiplicity) in the other.

This class of events should have a very clear topology, and no standard model background. One source of background would be from a massive fourth generation $\nu^{\prime}$, which could be produced by W exchange in $\mathrm{e}^{+} \mathrm{e}^{-} \rightarrow \nu^{\prime} \nu_{\mathrm{e}}$ and produce a similar signal. If such wonderful physics were to occur, separating the origins would not be difficult. For example, a fourth generation $\nu^{\prime}$ would also have decays $\mathrm{e}^{-} \pi^{+}$, $\mu^{-} \pi^{+}$, and it is produced by W exchange rather than sealar exchange so the angular distributions and energy distributions are different. A second source of "background" could come from $\mathrm{e}^{+} \mathrm{e}^{-} \rightarrow \tilde{\nu} \tilde{\nu}$, where one $\tilde{\nu}$ decays into $\nu \tilde{\gamma}$ and the other into a final state including charged particles. This kind of process is being carefully studied by Barnett, Haber, and Lackner [12].

Some of the particles $\left(\tilde{\gamma}_{2}, \nu^{\prime} \cdots\right)$ will have typical weak lifetimes that could show up in high resolution detectors, so that could be an additional clue.

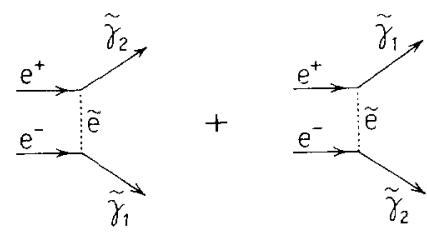

(a)

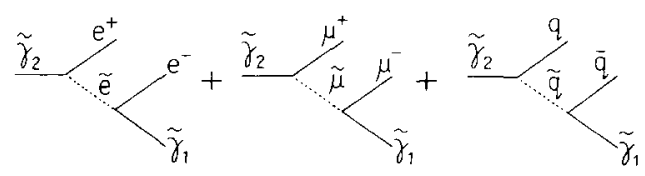

(b)

Fig. 4. Production of $\dot{\gamma}_{2}$ and $\tilde{\gamma}_{1}$ and decay of $\tilde{\gamma}_{2}$. 
The cross section for $\mathrm{e}^{+} \mathrm{e}^{-} \rightarrow \tilde{\gamma}_{2} \tilde{\gamma}_{1}$ is of the order of a typical weak cross section. At the vertices in fig. $4 \mathrm{a}$ one has a factor of $e \times g \times$ mixing angle factors. Then the cross section is about

$$
\sigma \simeq\left(\lambda_{1} \lambda_{2}\right)^{2} \alpha\left(G_{\mathrm{F}} m_{\mathrm{W}}^{2}\right) \frac{s}{m_{\tilde{\mathrm{e}}}^{4}},
$$

where $\lambda_{1}$ and $\lambda_{2}$ are the mixing angle factors: typically $0.1 \leqslant \lambda_{1} \lambda_{2} \leqslant 0.7$. Then for $s \simeq 1000 \mathrm{GeV}^{2}$ and the $m_{\tilde{\mathrm{e}}} \approx m_{\mathrm{W}}$,

$$
\sigma \simeq 20\left(\lambda_{1} \lambda_{2}\right)^{2} \mathrm{pb} .
$$

For $\lambda_{1} \lambda_{2} \geqslant 0.1, \sigma \geqslant 0.2 \mathrm{pb}$. An accelerator running at $10 \mathrm{pb}^{-1} /$ week would get $\geqslant 2$ events/week of $\tilde{\gamma}_{2} \tilde{\gamma}_{1}$ production, and it could be that $\lambda_{1} \lambda_{2}$ is much larger than 0.1 .

About $\frac{1}{5}$ of the $\tilde{\gamma}_{2}$ decay into $\mathrm{e}^{+} \mathrm{e}^{-} \tilde{\gamma}_{1}, \frac{1}{5}$ into $\mu^{+} \mu^{-} \tilde{\gamma}_{1}, \frac{1}{5}$ into $\tau^{+} \tau^{-} \tilde{\gamma}_{1}$, and $\frac{2}{5}$ into $\mathrm{q} \overline{\mathrm{q}} \tilde{\gamma}_{1}$. In all cases the basic signature is nothing in one hemisphere, with no standard model background.

With data soon available at $\mathrm{e}^{+} \mathrm{e}^{-}$colliders it should be possible to either detect such a state, or exclude it up to $m\left(\tilde{\gamma}_{2}\right)+m\left(\tilde{\gamma}_{1}\right) \leqslant \sqrt{ } s$ for a large range of couplings. Many actual models will occur in this region, with $m\left(\tilde{\gamma}_{1}\right)$ a few $\mathrm{GeV}, m\left(\tilde{\gamma}_{2}\right)<$ $30-40 \mathrm{GeV}$, and a product of couplings $\lambda_{1} \lambda_{2}$ of order 0.3 . If a signal is not found the impact on models may be very large. There has been one reported search, by the JADE collaboration [13], with no signal. However, it was optimized for $\mathrm{e}^{+} \mathrm{e}^{-} \rightarrow \nu^{\prime} \nu_{\mathrm{e}}, \nu^{\prime} \rightarrow \mathrm{eq} \overline{\mathrm{q}}$, so events with less than $40 \%$ of $\sqrt{ } s$ visible were cut, and those contain most of our signal.

Another signal of use may be

$$
\mathrm{e}^{+} \mathrm{e}^{-} \rightarrow \tilde{\gamma}_{2}+\tilde{\gamma}_{2}
$$

with both sides decaying to $\ell^{+} \ell^{-} \tilde{\gamma}_{1}$. This has been discussed in ref. [2]. Because of the reduced kinematic range, requiring $m\left(\tilde{\gamma}_{2}\right)<\frac{1}{2} \sqrt{ } s$, and the reduced signal due to the branching ratio effects, with $\left.\left(\operatorname{BR}\left(\mathrm{e}^{+} \mathrm{e}^{-} \tilde{\gamma}_{1}\right) \operatorname{BR}\left(\mu^{+} \mu^{-} \tilde{\gamma}_{1}\right)\right) \approx \frac{1}{9}\right)^{2}$, this may be less sensitive then the case we have discussed above, but it is also worth looking for.

We appreciate very much conversations with $R$. Thun, D. Meyer, S. Ting, D. Burke, D. R. T. Jones, and especially S. Raby. This research was supported in part by the US DoE.

\section{References}

[1] P. Fayet, Proc. of the 21st Int. High energy physics Conf., Paris, July 1982

[2] J. Ellis and G.G. Ross, Phys. Lett. 117B (1982) 397

[3] R. Arnowitt, A.H. Chamseddine and Pran Nath, Phys. Rev. Lett. 50 (1983) 232

[4] S. Weinberg, Phys. Rev. Lett. 50 (1983) 387

[5] L.E. Ibáñez, Nucl. Phys. B218 (1983) 514

[6] J. Polchinski, private communication 
[7] R. Barbieri, S. Ferrara and C.A. Savoy, Phys. Lett. 119B (1982) 343;

E. Cremmer, S. Ferrara, L. Girardello and A. Van Proeyen, CERN preprint TH-3312 (1982); Nucl. Phys. B212 (1983) 413;

H.P. Nilles, M. Srednicki and D. Wyler, CERN preprint TH-3432;

E. Cremmer, P. Fayet and L. Girardello, Ecole Normale Superieure, preprint LPTENS 82/30

[8] J.-M. Frère, D.R.T. Jones and S. Raby, Nucl. Phys. B222 (1983)

[9] Kwong H. Lau, Particle searches in $\mathrm{e}^{+} \mathrm{e}^{-}$experiments at PEP and PETRA, SLAC-PUB-3001, Oct. 1982

[10] B. Adeva et al., Phys. Rev. Lett. 48 (1982) 967

[11] W. Bartel et al., Z. Phys. C6 (1980) 295

[12] H. Haber, private communication, to be published

[13] JADE Collaboration, A Search for the new leptons at PETRA, DESY 83-002 Jan. 83 